\title{
An epidemiological study of isolated split hand/foot in Hungary, 1975-1984
}

\author{
A E Czeizel, M Vitéz, I Kodaj, W Lenz
}

\begin{abstract}
A population based and validated 10 year cohort of 94 cases with split hand/foot born in Hungary, 1975-1984 was evaluated. This type of congenital limb deficiency was relatively frequently $(43 \%)$ associated with non-limb defects. Fiftyfour cases with isolated split hand/foot are evaluated in this paper. A single limb was affected in $78 \%$ of cases. The upper limbs were 21 times more frequently affected in unimelic cases with a right sided predominance and male excess. Casecontrol analysis indicated intrauterine growth retardation and lower socioeconomic status of parents of cases. Family study showed six familial cases with autosomal dominant inheritance among 152 first and 452 second degree relatives. All familial cases were males.

(f Med Genet 1993;30:593-6)
\end{abstract}

Split hand/foot (SHF) is a congenital limb deficiency of the central rays of the upper or lower limbs or both. The following definition of SHF was used in this study: absence of central digits with or without absence of central metacarpal/metatarsal bones, usually associated with syndactyly of other digits. The socalled 'central' rays represent the second, third, and fourth rays which are embryologically differentiated at a different time from the pre- and postaxial rays. The birth prevalence of cases of SHF was 0.06 per 1000 total births in Hungary in 1975 to $1984 .{ }^{1}$ This rate is in agreement with previous ${ }^{2}$ and recent publications. $^{3}$

Three kinds of differentiation were performed in cases of SHF. First, SHF was separated into isolated (SFH without non-limb defect) and multiple (SHF associated with nonlimb defect) categories. ${ }^{4}$ Second, typical and atypical SHF were differentiated in isolated cases. ${ }^{5}$ Typical cases were in general multimelic, that is, more than one limb was involved, and frequently familial. Atypical cases were unimelic and sporadic and, in addition, the remaining marginal rays were always hypoplastic and the cleft may have had rudimentary digits. Third, there are two main subtypes of typical isolated SHF. ${ }^{2}$ (1) Lobster claw is characterised by a cone shaped cleft tapering proximally and dividing the hand/foot into two parts which can be opposed like lobster claws. In the mildest forms the third finger or toe is absent but the corresponding metacarpal/ metatarsal bone is almost normal. (2) Monodactyly is characterised by deficiency of the central and radial-tibial axes, that is, digits 1 to 4 , such that there is no cleft formation and only one digit, usually the fifth, is present. ${ }^{6}$

In this paper the findings of a case-control epidemiological study on a population based and validated 10 year cohort (derived from 1575904 births) of 54 cases with isolated SHF are presented. The data concerning multiple SHF cases will be published in another paper. ${ }^{7}$

\section{Materials and methods}

Cases were selected from the Hungarian Congenital Abnormality Registry ${ }^{8}$ and three other sources: records of paediatric orthopaedic surgeries where cases with congenital limb deficiencies are treated in Hungary, genetic counselling clinics, and the financial support services for the families of handicapped children. The total number of cases with SHF was 94. Patients were examined in our Department or at their home and the epidemiological data were obtained by questionnaire at a family interview. Of 54 cases with isolated SHF, 49 $(90 \cdot 7 \%)$ were examined personally. Detailed medical documents (for example, necropsy reports) were evaluated for five additional cases (including one stillbirth). Two cases $(3 \cdot 7 \%)$ with unimelic SHF could not be contacted because their new addresses were unknown, so epidemiological and familial data were not available. Of 52 mothers and fathers and 48 sibs, $49(94 \cdot 2 \%), 48(92 \cdot 3 \%)$, and $44(91 \cdot 7 \%)$, respectively, were examined, while the remainder and 452 second degree (grandparents, uncles, and aunts) and 263 third degree (first cousins) relatives were evaluated on the basis of information obtained at the family interview and available medical documents.

Controls were selected from the records of the Birth Registry of the Central Statistical Office and matched by sex, year of birth, and place of residence of the family of cases. Controls were visited at home by the regional social nurses and the questionnaire was completed by the mothers using the same method as for cases. Each case had two controls in order to prevent the loss of cases owing to nonparticipation of control families. If the first control did not cooperate, the second (13\%) was visited.

$\chi^{2}$, Fisher exact, and $U$ tests, and multivariate analyses ${ }^{9}$ were used to test the statistical significance of the study variables. Three categories of education $(\leqslant 7,8-11$, and $\geqslant 12$ school grades) and two categories of pregnancy (wanted, unwanted) were differentiated as confounders. 
Demographic and reproductive factors in the case and control groups.

\begin{tabular}{|c|c|c|c|}
\hline Factors & $\begin{array}{c}\text { Cases } \\
(\mathrm{n}=52)\end{array}$ & $\begin{array}{l}\text { Controls } \\
(\mathrm{n}=52)\end{array}$ & p \\
\hline \multicolumn{4}{|l|}{ Demographic } \\
\hline Birth weight: (g) (mean [SD]) & $3000[543]$ & 3359 [491] & $<0.005$ \\
\hline Low birth weight: $\leqslant 2500 \mathrm{~g}(\%)$ & $15 \cdot 4$ & 3.8 & $<0.01$ \\
\hline Gestational age: week (mean [SD]) & $39 \cdot 1[1 \cdot 6]$ & $39 \cdot 6[1 \cdot 6]$ & $>0.05$ \\
\hline & $25 \cdot 3[5 \cdot 2]$ & $25 \cdot 8[4 \cdot 4]$ & $>0.05$ \\
\hline $\begin{array}{l}\text { Birth order (mean [SD]) } \\
\text { Maternal education: No of school grades (mean [SD]) }\end{array}$ & $1.85[1.09]$ & $1.69[0.9]$ & $>0.05$ \\
\hline \multirow{2}{*}{\multicolumn{4}{|c|}{ Employment of mothers (No, \%) }} \\
\hline Professional & & & \\
\hline Clerk & $\begin{array}{l}713.5 \\
713.5\end{array}$ & $\begin{array}{r}15 \\
815 \cdot 4\end{array}$ & \multirow{5}{*}{$<0.01$} \\
\hline Skilled & $2140 \cdot 4$ & $2344 \cdot 2$ & \\
\hline Semiskilled & $917 \cdot 3$ & 11.9 & \\
\hline Unskilled & 11.9 & $2 \quad 3.8$ & \\
\hline Housewife & 713.5 & $\begin{array}{ll}35 \cdot 8 \\
5\end{array}$ & \\
\hline $\begin{array}{l}\text { Paternal age: year (mean }[S D] \text { ) } \\
\text { Paternal education: No of school grades (mean [SD]) }\end{array}$ & $27 \cdot 8[5 \cdot 3]$ & $28 \cdot 0[5 \cdot 3]$ & $>0.05$ \\
\hline \multirow{2}{*}{\multicolumn{3}{|c|}{ Reproduction }} & \\
\hline Unmarried (No, \%) & & & \\
\hline Unwanted pregnancies (No, \%) & $\begin{array}{ll}11 & 21 \cdot 2 \\
1325 \cdot 0\end{array}$ & $815 \cdot 4$ & $>0.03$ \\
\hline Infertility problem (No, \%) & $35 \cdot 7$ & 11.9 & $>0.05$ \\
\hline
\end{tabular}

\section{Results}

\section{MORPHOLOGY}

Of 54 isolated SHF cases, one limb was affected in $42(77.8 \%)$ cases. Upper limb predominance was obvious (40:2). Of 40 unimelic cases with upper limb involvement, in seven cases only the fingers were affected, while in 33 cases the metacarpals and fingers were affected. Two cases with unimelic upper limb involvement had monodactyly, one male with definite monodactyly and one female with probable monodactyly. There was a right sided (25:15) and male (27:13) excess. Two unimelic boys with lower limb involvement had their left sides affected. Of 54 isolated and 42 unimelic SHF cases, $33(61 \cdot 1 \%$ and $78.6 \%)$ had an atypical manifestation. One boy had left lower limb involvement, while all other atypical cases had an upper limb defect. The ratio of right to left and male to female was $17: 15$ and 19:13, respectively, in atypical upper limb SHF cases. Girls with unimelic upper limb involvement had only atypical SHF. Of 29 unimelic boys, nine $(31.0 \%)$ had typical SHF. One boy had left lower limb involvement, while there was right side predominance in the upper limb (7:1). Two limbs were affected with typical SHF in eight $(14.8 \%)$ cases (metacarpal-metacarpal in five boys; metatarsalmetatarsal in one boy; toes-toes in one girl; metatarsal-toes in one boy). Thus, bilateral instead of ipsilateral manifestation is characteristic. The male excess of affected upper limbs is also obvious. One boy $(1 \cdot 8 \%)$ had involvement of three limbs: left hand (absence of 3rd finger) and both feet (absence of toes 34). All limbs were affected with typical SHF in three girls $(5 \cdot 6 \%)$. Of 21 typical SHF cases, 12 $(57 \cdot 1 \%)$ were multimelic and $18(85 \cdot 7 \%)$ occurred in boys. All the 14 cases with typical SHF restricted to upper limbs were male.

DEMOGRAPHIC FINDINGS AND REPRODUCTION Epidemiological data were available in 52 cases and controls (table). A lower mean birth weight and a higher rate of low birth weight were seen in the cases. The gestational age was shorter in the cases than in the control group, but not significantly so. Thus, the lower birth weight can be explained mainly by intrauterine growth retardation.

There was no significant difference in the birth order or maternal or paternal ages between the case and control groups. The years of completed school grades were fewer in the mothers and fathers of cases and the employment status of the mothers of cases was in agreement with their lower level of education.

There was no significant difference in the reproductive variables between the case and control groups.

\section{TERATOGENIC AND MATERNAI FACTORS}

The exposure to potential teratogens (physical trauma: $3 v 1$; serious psychosocial stress: $4 v$ 5 ; diagnostic $x$ ray involvement of the abdominal or pelvic region or both: $0 v 1$; radiotherapy: $1 v 0$ ) in the pregnancies of case and control mothers were not significantly different. Of 52 case and control mothers, 34 $(65 \cdot 3 \%)$ and $32(59 \cdot 6 \%)$ took medication. Occupational (radiation, chemical, infection, etc) hazards did not show any significant difference for the 36 case and 37 control mothers who worked during pregnancy.

The number of females who smoked during pregnancy $(18 v 9)$ was higher in the case group than in the control group, particularly for heavy smokers $(8 v 0)$. However, once adjustment was made for the educational level of the mothers and the proportion of unwanted pregnancies, these differences were not significant. There was a higher rate of alcohol intake in the control group ( $3 v 10)$.

The total rate of acute maternal disorders during pregnancy was significantly higher in the case group $(20: 38.5 \% v 4: 7 \cdot 7 \%)$ $(p=0.0004)$ because of acute infections of the respiratory system, mainly influenza $(13 v 3)$ (Fisher $p=0.008$ ). The occurrence of influenza in the first, second, and third trimesters of pregnancy was $5: 2,6: 1$, and $2: 0$, respectively. Thus, the majority of attacks of influenza occurred after the critical period for SHF.

There was no significant difference 


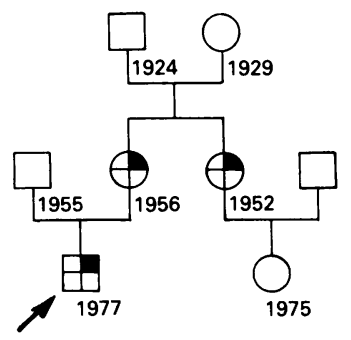

$343 / 1978$
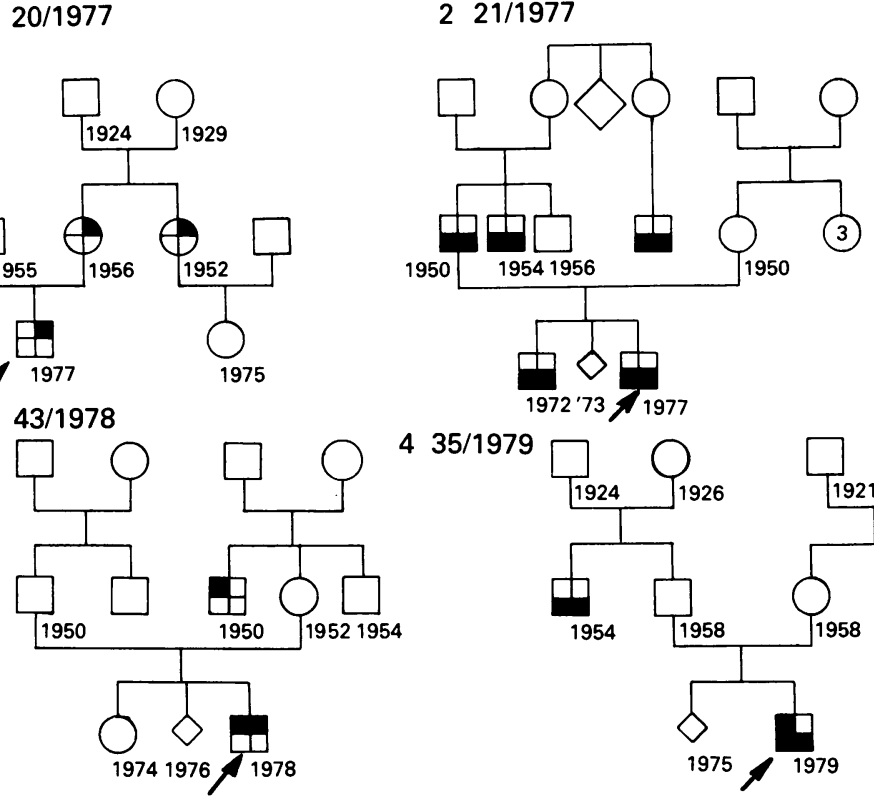

$435 / 1979$
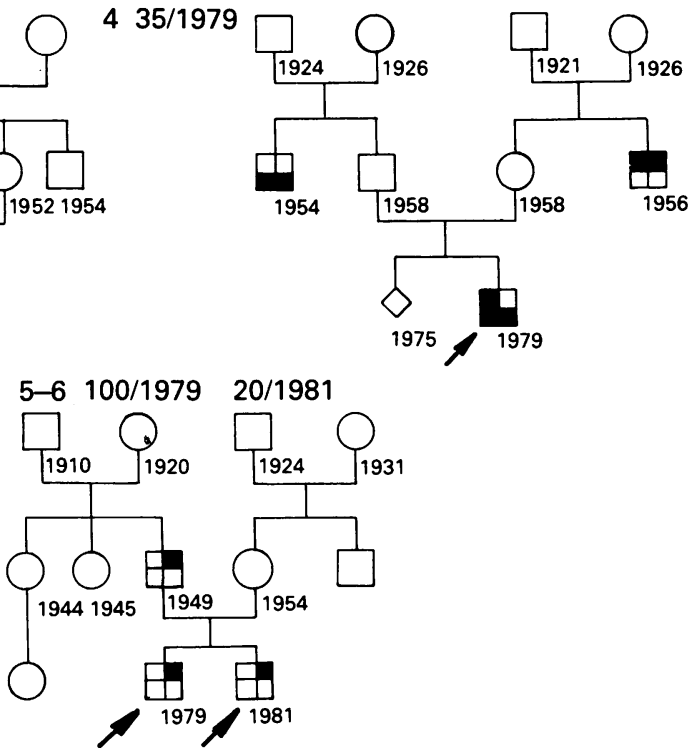

Figure 1 Pedigrees of familial cases.

between the case and control groups, in the frequency of different chronic maternal disorders.

\section{FAMILY STUDY}

Of 52 cases, four had affected first degree relatives (fig 1). In two families two brothers were affected, but only one family had both brothers as index cases. All familial cases were male. There were $3.9 \%$ of parents affected (4/ 104 ) and $7 \cdot 7 \%$ of both parents (4/52), compared to $6.3 \%$ of sibs (3/48).

One case, a boy (20/1977), had an absent right third finger probably with some fusion of fingers 3 and 4 . His mother had pronounced camptodactyly of fingers 4 and 5 and partial syndactyly between fingers 2 and 3 as well as 4 and 5 in the right hand. The sister of the mother had absence of fingers and metacarpals 3-5 with camptodactyly and hypoplastic phalanges of the second finger on her right hand. The feet and the left hand were not involved in the three affected members of this family. Another case, also a boy (21/1977), had bilateral split feet, absence of toes 2 to 3 on the right and absence of the second toe and partial absence of the third toe on the left while his brother had a nearly identical SHF and his father had a similar SHF comprising absence or severe hypoplasia of the middle and terminal phalanges of toes 2 to 5 with syndactyly on both sides. The paternal grandparents were stated to be unaffected; however, one brother and a maternal male cousin of the father had a

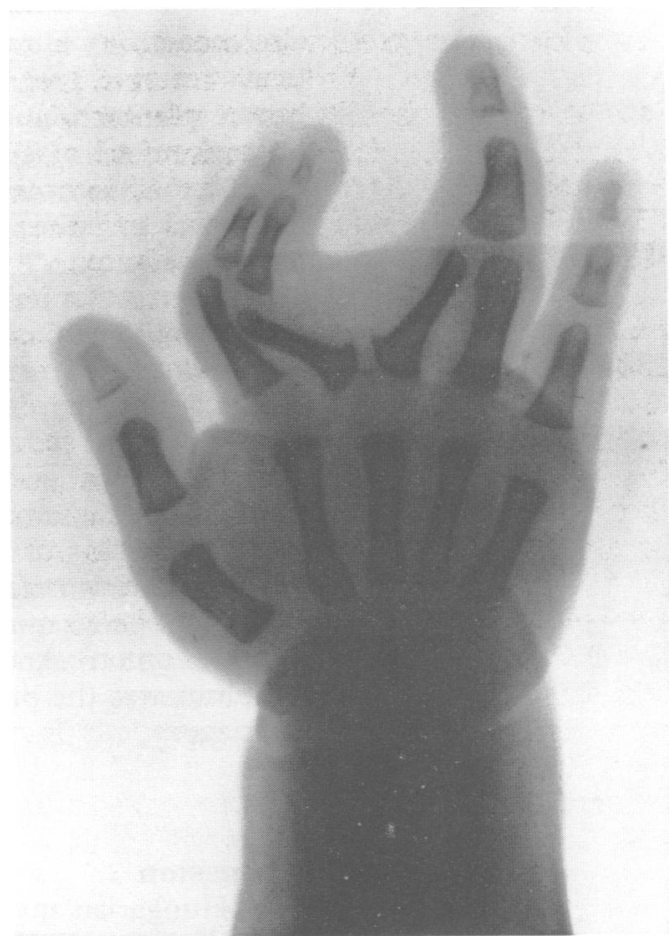

Figure 2 Radiograph of right hand of case 100/1979.

similar SHF in their feet. Two brothers (100/ 1979 and 20/1981) had a nearly identical right unimelic SHF (fig 2); their father had severe hypoplasia of the right third finger which was later removed. The maternal grandparents were not affected.

It is worth mentioning that the previously described three girls with four affected limbs, and the one boy with three affected limbs, had no positive family history. However, we cannot exclude the possibility that these cases were the consequence of a new mutation, or they may have had a non-penetrant parent.

SHF occurred in the second degree relatives of four cases (fig 1); however, two second degree relatives belonged to the previously described families $(20 / 1977$ and $21 / 1977)$. The fifth familial case occurred in the uncle of a case $(43 / 1978)$ with a defect of the third ray in both hands. The maternal uncle had a unilateral left split hand. No radiological defect could be detected in the mother of this case. In the sixth familial case $(35 / 1979)$, the boy had a SHF of the left hand and both feet. His paternal uncle was affected with bilateral split feet (partial absence of toes 2 and 3 with syndactyly between toes 3 and 4). His maternal uncle was reported to have four fingers only in both hands but a personal examination could not be carried out. The mother and father of this case have no SHF but both are deaf. This case is also affected with deafness, thus the co-occurrence of congenital deafness and split hand and foot syndrome cannot be excluded. Of 263 cousins, none had SHF. Thus, of 52 cases, six $(11.5 \%)$ were familial taking into consideration four cases with 'regular' dominant and two cases with 'irregular' dominant inheritance.

In evaluating occurrence in sibs, the outcomes of previous and subsequent pregnancies may be important if there is prenatal loss of 
affected fetuses. However, a higher rate of fetal death (ectopic pregnancy, miscarriages, stillbirth) was not found in the case group. The lower number of subsequent pregnancies of case mothers is noteworthy (10v 42) and may indicate a negative effect of SHF on future family planning.

The rate of other congenital abnormalities $(4.6 \%)$ in the first degree relatives of cases corresponds well with the expected figure based on the recorded Hungarian data (about $4 \%$ ). However, this figure was distorted by a familial aggregation of patella aplasia in three members of the same family.

Of 52 parents of cases, consanguinity was mentioned in two cases. One case was reported to be the consequence of incest (fatherdaughter union). Another case with four affected limbs was the product of a second cousin marriage.

\section{Discussion}

This Hungarian material represents the first study on isolated SHF cases in a validated and population based data set. The main morphological characteristics of SHF are as follow.

(1) SHF is seen relatively frequently with non-limb defects. Of 94 cases, $40(42 \cdot 6 \%)$ were multiple. Thus, the ratio of isolated and multiple cases is 1:0.74.

(2) About $78 \%$ of isolated SHF cases were unimelic.

(3) SHF occurs more frequently in the upper limb. The ratio of upper to lower limbs was $21: 1$ in unimelic cases.

(4) About $61 \%$ of all cases were atypical SHF.

(5) There was a right sided predominance in cases with typical SHF, while the side involvement seemed to be random in atypical cases.

(6) There was a male excess in cases with involvement of one or two limbs and all cases with typical SHF restricted to upper limbs were male, while three cases involving four limbs were female. All girls with unimelic manifestation had atypical SHF.

(7) Monodactyly of the hand occurred in one or two cases.

First, the familial nature of several cases of isolated SHF with autosomal dominant inheritance should be mentioned. Of six familial cases, however, two apparently had a nonpenetrant parent and four other maternal grandparents were presumably carriers (fig 1). This phenomenon and different expression of the same gene is well known in the SHF. ${ }^{2}$ The multimelic cases included a higher rate of familial cases compared with unimelic cases (3/ $12=25 \%$ v $3 / 40=7 \cdot 5 \%$ ); however, autosomal dominant inheritance may also occur in typical unimelic cases. Thus, morphological classifi- cation is not a great help from the aetiological aspect. Another important point is the male excess, especially in familial $(100 \%)$ cases (fig 1). This cannot be explained by prenatal selection because SHF is not lethal and the fetal death rate was not higher in the previous and subsequent pregnancies of their mothers. Ahmad et $a l^{10}$ reported a Pakistani kindred in which 36 members in seven generations had SHF. The full expression of SHF was present in 33 males and three females. Other females showed a distinctly milder expression of SHF. The distribution of affected members in the pedigree was considered to be compatible with $\mathrm{X}$ linked inheritance. Hemizygous males and presumably homozygous females exhibit typical SHF, whereas only some of the obligatory heterozygous females show the milder expression. However, in our study father to son transmission occurred in two families. A disturbed segregation ratio found in the families of SHF cases has been explained by germinal selection, gonadal mosaicism, premutation of an autosomal dominant gene, or cosegregation of an epistatic gene linked to the gene for SHF. ${ }^{1112}$

Familial cases, however, represent only a proportion of isolated SHF cases. The sporadic and unimelic manifestation with nearly exclusive involvement of the upper limbs, the intrauterine growth retardation, and the lower socioeconomic status of the parents seem to be characteristic of the remaining cases, mainly atypical SHF. Some of these findings are in agreement with the vascular disruption hypothesis. ${ }^{13}$

1 Czeizel A, Vitéz M, Kodaj I, Lenz W. Birth prevalence of different congenital limb deficiency types in a populationbased revised Hungarian material 1975-1984. Acta Morphol Hung 1991;39:229-37.

2 Birch-Jensen AI. Congenital deformities of the upper extremities. Copenhagen: Munksgaard, 1949.

3 ICBDMS: Annual Report, 1982. International Clearinghouse for Birth Defects Monitoring Systems, Stockholm 1984

4 Czeizel A, Telegdi L, Tusnády G. Multiple congenital abnormalities. Budapest: Akadémiai Könyvkiadó, 1988.

5 Lange M. Grundsatzlicher über die Beurteilung der Entstehung und Bewertung atypischer Hand- und Fussmissbildungen. $Z$ Orthop 1937;66:80-8.

6 Bujdosó G, Lenz W. Monodactylous splithand-splitfoot: a malformation occurring in three distinct genetic types. malformation occurring in thr

7 Evans JA, Vitéz M, Czeizel A. Congenital abnormalities associated with limb reduction deficiencies: a populationassociated with limb reduction deficiencies: a populationpress)

8 Czeizel A. The activities of the Hungarian Centre for Congenital Anomaly Control. WHO Stat Rep Quar 1988;41:219-27.

9 Holford TR, White C, Kelsey JL. Multivariate analysis for matched case-control studies. Am $\mathcal{f}$ Epidemio 1978;107:245-2.

10 Ahmad M, Abbas H, Haque S, Flatz G. X-chromosomally irherited split-hand/split foot anomaly in a Pakistan kindred. Hum Genet 1987;75:169-73.

11 Stevenson AC, Jennings LM. Ectrodactyly-evidence in favour of a disturbed segregation in the offspring of affected males. Ann Hum Genet 1960;24:89-96.

12 Spranger $M$, Schapera J. Anomalous inheritance in kindred with split hand, split foot malformations. Eur $f$ Pediatr 1988;147:202-5.

13 Hoyme HE, van Allen MI, Jones KL. The vascular pathogenesis of some sporadically occurring limb defects. Semin Perinatol 1983;7:299-306. 